Wojciech Kucharz

\title{
Cycles on algebraic models of smooth manifolds
}

Received March 3, 2007 and in revised form June 15, 2007

Abstract. Every compact smooth manifold $M$ is diffeomorphic to a nonsingular real algebraic set, called an algebraic model of $M$. We study modulo 2 homology classes represented by algebraic subsets of $X$, as $X$ runs through the class of all algebraic models of $M$. Our main result concerns the case where $M$ is a spin manifold.

Keywords. Real algebraic sets, algebraic cohomology classes, algebraic models

\section{Introduction}

Let $X$ be a compact nonsingular real algebraic set (in $\mathbb{R}^{n}$ for some $n$ ). A cohomology class in $H^{k}(X, \mathbb{Z} / 2)$ is said to be algebraic if the homology class Poincare dual to it can be represented by an algebraic subset of $X$. The set $H_{\text {alg }}^{k}(X, \mathbb{Z} / 2)$ of all algebraic cohomology classes in $H^{k}(X, \mathbb{Z} / 2)$ is a subgroup, while the direct sum $H_{\mathrm{alg}}^{*}(X, \mathbb{Z} / 2)$ of the $H_{\mathrm{alg}}^{k}(X, \mathbb{Z} / 2)$, for $k \geq 0$, forms a subring of the cohomology ring $H^{*}(X, \mathbb{Z} / 2)$. Early papers dealing with algebraic cohomology (or homology) classes provided examples of $X$ with $H_{\text {alg }}^{*}(X, \mathbb{Z} / 2) \neq H^{*}(X, \mathbb{Z} / 2)$ (cf. [1, 5, 6, 14, 19, 20]). The reader can find a survey of properties and applications of $H_{\mathrm{alg}}^{*}(-, \mathbb{Z} / 2)$ in [11].

Every compact smooth (of class $\mathcal{C}^{\infty}$ ) manifold $M$ is diffeomorphic to a nonsingular real algebraic set, called an algebraic model of $M$ (cf. [23]; see also [7, Theorem 14.1.10] and, for a weaker but influential result, [18]). The following question is a challenging problem: How does the ring $H_{\mathrm{alg}}^{*}(X, \mathbb{Z} / 2)$ vary as $X$ runs through the class of algebraic models of $M$ ? This paper provides partial answers. Due to technical difficulties it is easier to describe how the group $H_{\mathrm{alg}}^{k}(X, \mathbb{Z} / 2)$ varies for a fixed $k$. Results of this type are in [8] for $k=1$, in [10] for $k=2$, and in [16] for $k \geq 3$. If $k \geq 2$ and especially if $k \geq 3$ they are far from complete.

We say that a subring $A$ of $H^{*}(M, \mathbb{Z} / 2)$ is algebraically realizable if there exist an algebraic model $X$ of $M$ and a smooth diffeomorphism $\varphi: X \rightarrow M$ with $\varphi^{*}(A) \subseteq H_{\mathrm{alg}}^{*}(X, \mathbb{Z} / 2)$. The original goal of several researchers was to show that the

W. Kucharz: Max-Planck-Institut für Mathematik, Vivatsgasse 7, 53111 Bonn, Germany, and Department of Mathematics and Statistics, University of New Mexico, Albuquerque, NM 87131-1141, U.S.A.; e-mail: kucharz@math.unm.edu

Mathematics Subject Classification (2000): 14P05, 14P25, 57R19 
whole ring $H^{*}(M, \mathbb{Z} / 2)$ is algebraically realizable, that is, $M$ has an algebraic model $X$ with $H_{\text {alg }}^{*}(X, \mathbb{Z} / 2)=H^{*}(X, \mathbb{Z} / 2)$ (such a conjecture, motivated by far-reaching potential applications, was explicitly stated in [1]). However, since the publication of [3] it has been known that for some manifolds $M$ this is impossible. An important algebraically realizable subring of $H^{*}(M, \mathbb{Z} / 2)$ is identified in [4, Theorem 4, Remark 8]. It is the subring $A(M)$ generated by the Stiefel-Whitney classes of all real vector bundles on $M$ together with the cohomology classes Poincare dual to the homology classes represented by all smooth submanifolds of $M$. A conjecture proposed in [3], and still open at the present time, suggests that every algebraically realizable subring of $H^{*}(M, \mathbb{Z} / 2)$ is contained in $A(M)$.

For us, certain subrings of $A(M)$ will play a crucial role. We say that a subring $A$ of $H^{*}(M, \mathbb{Z} / 2)$ is admissible if it is generated by the Stiefel-Whitney classes of some real vector bundles on $M$ and the cohomology classes Poincaré dual to the homology classes represented by some smooth submanifolds of $M$. Thus $A(M)$ is the largest admissible subring of $H^{*}(M, \mathbb{Z} / 2)$. However, in general, not every subring of $A(M)$ is admissible. Given any subring $A$ of $H^{*}(M, \mathbb{Z} / 2)$, we set $A^{k}=A \cap H^{k}(M, \mathbb{Z} / 2)$. As usual, we denote by $w_{i}(M)$ the $i$ th Stiefel-Whitney class of $M$. Recall that $M$ is called a spin manifold if $w_{1}(M)=0$ and $w_{2}(M)=0$.

Theorem 1.1. Let $M$ be a compact connected spin manifold. Assume that $\operatorname{dim} M \geq 7$ and the group $H_{i}(M, \mathbb{Z})$ has no 2-torsion for $i=1,2$. Then for any admissible subring A of $H^{*}(M, \mathbb{Z} / 2)$, there exist an algebraic model $X$ of $M$ and a smooth diffeomorphism $\varphi: X \rightarrow M$ satisfying

$$
\varphi^{*}(A) \subseteq H_{\mathrm{alg}}^{*}(X, \mathbb{Z} / 2) \quad \text { and } \quad \varphi^{*}\left(A^{k}\right)=H_{\mathrm{alg}}^{k}(X, \mathbb{Z} / 2) \quad \text { for } k=0,1,2,3 .
$$

As we mentioned above, some results of this type have already been known. More precisely, for $M$ and $A$ as in Theorem 1.1, given $k=1$ or $k=2$, one can find an algebraic model $X_{k}$ and a smooth diffeomorphism $\phi_{k}: X_{k} \rightarrow M$ with $\phi_{k}^{*}\left(A^{k}\right)=H_{\text {alg }}^{k}\left(X_{k}, \mathbb{Z} / 2\right)$ (cf. [8, 10]; see also [16] for $k=3$, but with different, somewhat artificial, assumptions). Thus the main contribution of Theorem 1.1 is the existence, under natural assumptions, of $X$ and $\phi$ satisfying $\phi^{*}\left(A^{k}\right)=H_{\text {alg }}^{k}(X, \mathbb{Z} / 2)$ simultaneously for $k=1,2,3$ ( $k=0$ being trivial). Our more general result, Theorem 2.4 in Section 2, concerns arbitrary $k$, but requires rather technical conditions on $M$ and $A$. In view of Lemma 2.5, these technical conditions disappear for $k \leq 3$, and thus we get Theorem 1.1. It seems, however, that a completely new idea is needed in order to obtain interesting results for $k>3$.

Theorem 1.1 is particularly nice in dimension 7,8 or 9 .

Corollary 1.2. Let $M$ be a compact connected spin manifold of dimension $m$, where $m=7,8$, or 9 . Assume that the group $H_{i}(M, \mathbb{Z})$ has no 2 -torsion for $i=1, \ldots, m-5$. Then for any subring $A$ of $H^{*}(M, \mathbb{Z} / 2)$, there exist an algebraic model $X$ of $M$ and $a$ smooth diffeomorphism $\varphi: X \rightarrow M$ satisfying

$$
\varphi^{*}(A) \subseteq H_{\mathrm{alg}}^{*}(X, \mathbb{Z} / 2) \quad \text { and } \quad \varphi^{*}\left(A^{k}\right)=H_{\mathrm{alg}}^{k}(X, \mathbb{Z} / 2) \quad \text { for } k=0,1,2,3 .
$$


It suffices to prove that under the assumptions of Corollary 1.2, every subring of $H^{*}(M, \mathbb{Z} / 2)$ is admissible. The latter fact easily follows from known results (see the next section). One can also drop the assumption about the dimension of $M$ in Corollary 1.2 , provided that the topology of $M$ is not too complicated (cf. Example 2.6).

For manifolds which are not necessarily spin, we have the following result.

Theorem 1.3. Let $M$ be a compact connected smooth manifold. Assume that $\operatorname{dim} M=$ $m \geq 5$ and the group $H_{m-2}(M, \mathbb{Z})$ has no 2-torsion. Then for any admissible subring $A$ of $H^{*}(M, \mathbb{Z} / 2)$, the following conditions are equivalent:

(a) There exist an algebraic model $X$ of $M$ and a smooth diffeomorphism $\varphi: X \rightarrow M$ satisfying

$$
\varphi^{*}(A) \subseteq H_{\text {alg }}^{*}(X, \mathbb{Z} / 2) \quad \text { and } \quad \varphi^{*}\left(A^{k}\right)=H_{\text {alg }}^{k}(X, \mathbb{Z} / 2) \quad \text { for } k=0,1,2 .
$$

(b) $w_{i}(M)$ is in $A^{i}$ for $i=1,2$.

If $\operatorname{dim} M=5$, then every homology class in $H_{d}(M, \mathbb{Z} / 2), d \geq 0$, can be represented by a smooth submanifold [22, Théorème II.26], and hence every subring of $H^{*}(M, \mathbb{Z} / 2)$ is admissible.

In order to compare the assumptions in Theorems 1.1 and 1.3, let us note that for any orientable compact smooth manifold $M$ of dimension $m$, the groups $H_{1}(M, \mathbb{Z})$ and $H_{m-2}(M, \mathbb{Z})$ have isomorphic torsion subgroups. Indeed, this follows from the Poincaré duality and the universal coefficient theorem for cohomology.

Theorems 1.1, 1.3 and Corollary 1.2 are proved in Section 2.

\section{Proofs and further results}

We will need some constructions from real algebraic geometry. Throughout this paper the term real algebraic variety designates a locally ringed space isomorphic to an algebraic subset of $\mathbb{R}^{n}$, for some $n$, endowed with the Zariski topology and the sheaf of $\mathbb{R}$-valued regular functions. Morphisms between real algebraic varieties will be called regular maps. Background material on real algebraic varieties and regular maps can be found in [7]. Every real algebraic variety carries also the Euclidean topology, which is determined by the usual metric topology on $\mathbb{R}$. Unless explicitly stated otherwise, all topological notions relating to real algebraic varieties will refer to the Euclidean topology.

The Grassmannian $\mathbb{G}_{n, r}$ of $r$-dimensional vector subspaces of $\mathbb{R}^{n}$ is endowed with a canonical structure sheaf which makes it into a real algebraic variety in the sense of this paper [7, Theorem 3.4.4] (an affine real algebraic variety according to the terminology used in [7]). Moreover, $\mathbb{G}_{n, r}$ is nonsingular and

$$
H_{\text {alg }}^{*}\left(\mathbb{G}_{n, r}, \mathbb{Z} / 2\right)=H^{*}\left(\mathbb{G}_{n, r}, \mathbb{Z} / 2\right)
$$

(cf. [7, Propositions 3.4.3 and 11.3.3]). The universal vector bundle $\gamma_{n, r}$ on $\mathbb{G}_{n, r}$ is algebraic. If $\xi$ is an algebraic vector bundle of rank $r$ on a real algebraic variety $X$ and if 
$n$ is a sufficiently large integer, then there is a regular map $f: X \rightarrow \mathbb{G}_{n, r}$ with $f^{*} \gamma_{n, r}$ algebraically isomorphic to $\xi$ (cf. [7, Theorem 12.1.7]). Here referring to algebraic vector bundles we follow [7], while in [4, 5, 6, 8, 9, 10] such bundles are called strongly algebraic.

Given a compact nonsingular real algebraic variety $X$, we define $\operatorname{Alg}^{k}(X)$ to be the set of all elements $u$ of $H^{k}(X, \mathbb{Z} / 2)$ for which there exist a compact nonsingular irreducible real algebraic variety $T$ (depending on $u$ ), two points $t_{0}$ and $t_{1}$ in $T$ and a cohomology class $z$ in $H_{\text {alg }}^{k}(X \times T, \mathbb{Z} / 2)$ such that

$$
u=i_{t_{1}}^{*}(z)-i_{t_{0}}^{*}(z)
$$

where for any $t$ in $T$, we let $i_{t}: X \rightarrow X \times T$ denote the map $i_{t}(x)=(x, t)$ for all $x$ in $X$. An equivalent description of $\operatorname{Alg}^{k}(X)$, which immediately implies that $\operatorname{Alg}^{k}(X)$ is a subgroup of $H_{\mathrm{alg}}^{k}(X, \mathbb{Z} / 2)$, is given in [15, 16]. The groups $H_{\mathrm{alg}}^{k}(-, \mathbb{Z} / 2)$ and $\operatorname{Alg}^{k}(-)$ have the expected functorial properties. If $f: X \rightarrow Y$ is a regular map between compact nonsingular real algebraic varieties, then the induced homomorphism $f^{*}: H^{*}(Y, \mathbb{Z} / 2) \rightarrow$ $H^{*}(X, \mathbb{Z} / 2)$ satisfies

$$
f^{*}\left(H_{\mathrm{alg}}^{k}(Y, \mathbb{Z} / 2)\right) \subseteq H_{\mathrm{alg}}^{k}(X, \mathbb{Z} / 2) \quad \text { and } \quad f^{*}\left(\operatorname{Alg}^{k}(Y)\right) \subseteq \operatorname{Alg}^{k}(X)
$$

(cf. [12, Section 5] or [6] for the former inclusion and [16] for the latter).

The following fact will be very useful.

Theorem 2.1 (cf. [15, Theorem 2.1]). Let $X$ be a compact nonsingular real algebraic variety. Then $\langle u \cup v,[X]\rangle=0$ for all $u$ in $\operatorname{Alg}^{k}(X)$ and $v$ in $H_{\text {alg }}^{\ell}(X, \mathbb{Z} / 2)$, where $k+\ell=$ $\operatorname{dim} X$.

As usual $\cup$ and $\langle$,$\rangle denote the cup product and scalar (Kronecker) product, while [X]$ stands for the fundamental class of $X$ in $H_{d}(X, \mathbb{Z} / 2), d=\operatorname{dim} X$.

We will also need some properties of $\operatorname{Alg}^{k}(-)$ for very specific real algebraic varieties. Let $B^{n}$ be a nonsingular irreducible real algebraic variety with precisely two connected components $B_{0}^{n}$ and $B_{1}^{n}$, each diffeomorphic to the unit $n$-sphere, $n \geq 1$. For example, one can take

$$
B^{n}=\left\{\left(x_{0}, \ldots, x_{n}\right) \in \mathbb{R}^{n+1} \mid x_{0}^{4}-4 x_{0}^{2}+1+x_{1}^{2}+\cdots+x_{n}^{2}=0\right\} .
$$

Let $B^{n}(d)=B^{n} \times \cdots \times B^{n}$ and $B_{0}^{n}(d)=B_{0}^{n} \times \cdots \times B_{0}^{n}$ be the $d$-fold products, and let $\delta: B_{0}^{n}(d) \hookrightarrow B^{n}(d)$ be the inclusion map. Then according to [16, Example 4.5],

$$
H^{q}\left(B_{0}^{n}(d), \mathbb{Z} / 2\right)=\delta^{*}\left(H^{q}\left(B^{n}(d), \mathbb{Z} / 2\right)\right)=\delta^{*}\left(\operatorname{Alg}^{q}\left(B^{n}(d)\right)\right)
$$

for all $q \geq 0$.

We now recall an important result from differential topology. All manifolds that appear here are without boundary. 
Theorem 2.3 ([13, (17.3)]). Let $P$ be a smooth manifold. Two smooth maps $f: M \rightarrow P$ and $g: N \rightarrow P$, where $M$ and $N$ are compact smooth manifolds of dimension $m$, represent the same bordism class in the unoriented bordism group $\mathcal{N}_{*}(P)$ if and only if for every nonnegative integer $q$ and every cohomology class $v$ in $H^{q}(P, \mathbb{Z} / 2)$, one has

$$
\left\langle w_{i_{1}}(M) \cup \cdots \cup w_{i_{r}}(M) \cup f^{*}(v),[M]\right\rangle=\left\langle w_{i_{1}}(N) \cup \cdots \cup w_{i_{r}}(N) \cup g^{*}(v),[N]\right\rangle
$$

for all nonnegative integers $i_{1}, \ldots, i_{r}$ with $i_{1}+\cdots+i_{r}=m-q$.

Let $M$ be a compact smooth manifold. For any positive integer $k$, we define $G^{k}(M)$ to be the subgroup of $H^{k}(M, \mathbb{Z} / 2)$ consisting of the cohomology classes $u$ satisfying

$$
\left\langle w_{i_{1}}(M) \cup \cdots \cup w_{i_{r}}(M) \cup u,[M]\right\rangle=0
$$

for all nonnegative integers $i_{1}, \ldots, i_{r}$ with $i_{1}+\cdots+i_{r}=m-k$.

A cohomology class $v$ in $H^{k}(M, \mathbb{Z} / 2), k \geq 1$, is said to be spherical provided $v=$ $f^{*}(c)$, where $f: M \rightarrow S^{k}$ is a continuous (or equivalently smooth) map from $M$ into the unit $k$-sphere $S^{k}$ and $c$ is the unique generator of the group $H^{k}\left(S^{k}, \mathbb{Z} / 2\right) \cong \mathbb{Z} / 2$. It is well known that $v$ is spherical if and only if the homology class Poincaré dual to $v$ can be represented by a smooth submanifold of $M$ with trivial normal vector bundle (cf. [22, Théorème II.2]). Denote by $S^{k}(M)$ the set of all spherical cohomology classes in $H^{k}(M, \mathbb{Z} / 2)$. It readily follows from the characterization of spherical cohomology classes recalled above that $S^{k}(M)$ is a subgroup of $H^{k}(M, \mathbb{Z} / 2)$ if $2 k \geq \operatorname{dim} M+1$.

For any smooth submanifold $N$ of $M$ of codimension $k$, we denote by $[N]^{M}$ the cohomology class in $H^{k}(M, \mathbb{Z} / 2)$ Poincaré dual to the homology class represented by $N$. As usual, if $\xi$ is a real vector bundle on $M$, then $w(\xi)$ and $w_{k}(\xi)$ will stand for, respectively, its total and $k$ th Stiefel-Whitney class. The total Stiefel-Whitney class of $M$ will be denoted by $w(M)$.

Given a collection $\mathcal{F}$ of real vector bundles on $M$ and a collection $\mathcal{G}$ of smooth submanifolds of $M$, we denote by $A(\mathcal{F}, \mathcal{G})$ the subring of $H^{*}(M, \mathbb{Z} / 2)$ generated by $w_{k}(\xi)$ and $[N]^{M}$ for all $\xi$ in $\mathcal{F}, k \geq 0$, and $N$ in $\mathcal{G}$. Since $H^{*}(M, \mathbb{Z} / 2)$ is a finite set, we may assume without loss of generality that the collections $\mathcal{F}$ and $\mathcal{G}$ are finite. By definition, any admissible subring of $H^{*}(M, \mathbb{Z} / 2)$ is of the form $A(\mathcal{F}, \mathcal{G})$.

Theorem 2.4. Let $M$ be a compact connected smooth manifold of dimension $m$. Let $\mathcal{F}$ be a collection of real vector bundles on $M$ and let $\mathcal{G}$ be a collection of smooth submanifolds of $M$. Assume that there is an integer $k_{0} \geq 2$ such that $2 k_{0}+1 \leq m$ and $\operatorname{codim}_{M} N \geq$ $k_{0}$ for all $N$ in $\mathcal{G}$. Then for the subring $A=A(\mathcal{F}, \mathcal{G})$ of $H^{*}(M, \mathbb{Z} / 2)$, the following conditions are equivalent:

(a) There exist an algebraic model $X$ of $M$ and a smooth diffeomorphism $\varphi: X \rightarrow M$ satisfying

and

$$
\varphi^{*}(A) \subseteq H_{\mathrm{alg}}^{*}(X, \mathbb{Z} / 2)
$$

$$
\varphi^{*}\left(A^{k}\right)=H_{\text {alg }}^{k}(X, \mathbb{Z} / 2) \quad \text { for all } k \text { with } k \leq k_{0} \text { and } G^{m-k}(M) \subseteq S^{m-k}(M) .
$$

(b) $w(M)$ is in $A$. 
Proof. If $Y$ is a compact nonsingular real algebraic variety, then $w(Y)$ is in $H_{\mathrm{alg}}^{*}(Y, \mathbb{Z} / 2)$ (cf. [6, 11, 12]), and hence (a) implies (b).

Assume that (b) holds. Let $\mathcal{F}=\left\{\xi_{1}, \ldots, \xi_{a}\right\}$ and $\mathcal{G}=\left\{N_{1}, \ldots, N_{b}\right\}$. For the use in a latter part of the proof, we modify each submanifold $N_{j}$, without affecting the cohomology class $\left[N_{j}\right]^{M}$, so as to obtain a new $N_{j}$ connected and nonorientable. This is possible since $M$ is connected and $\operatorname{codim}_{M} N_{j} \geq 2$. Indeed, the last inequality implies that if $U$ is an open subset of $M$ diffeomorphic to $\mathbb{R}^{m}$, then there is a smooth connected nonorientable submanifold $P_{j}$ of $M$ contained in $U$ and with $\operatorname{dim} P_{j}=\operatorname{dim} N_{j}$. Joining $P_{j}$ and the connected components of $N_{j}$ with tubes, we get the required modification of $N_{j}$.

By transversality, the submanifolds $N_{1}, \ldots, N_{b}$ can be chosen in general position. Hence in view of [4, Theorem 4, Remark 8], we may assume that $M$ is a nonsingular real algebraic variety, $N_{1}, \ldots, N_{b}$ are nonsingular Zariski closed subvarieties of $M$, and every topological real vector bundle on $M$ is isomorphic to an algebraic vector bundle. In particular, we may assume that $\xi_{1}, \ldots, \xi_{a}$ are algebraic vector bundles. Setting $r_{i}=$ rank $\xi_{i}$ and choosing a sufficiently large integer $n$, we can find a regular map $f_{i}: M \rightarrow$ $\mathbb{G}_{n, r_{i}}$ such that $\xi_{i}$ is isomorphic to $f_{i}^{*} \gamma_{n, r_{i}}$, and hence $w\left(\xi_{i}\right)=f_{i}^{*}\left(w\left(\gamma_{n, r_{i}}\right)\right)$. Therefore

$A$ is generated by $f_{i}^{*}\left(w_{k}\left(\gamma_{n, r_{i}}\right)\right)$ and $\left[N_{j}\right]^{M}, 1 \leq i \leq a, 1 \leq j \leq b, k \geq 0$.

Setting

$$
G=\mathbb{G}_{n, r_{1}} \times \cdots \times \mathbb{G}_{n, r_{a}} \text { and } f=\left(f_{1}, \ldots, f_{a}\right): M \rightarrow G,
$$

and making use of Künneth's theorem, we obtain

$$
f^{*}\left(H^{*}(G, \mathbb{Z} / 2)\right) \subseteq A .
$$

Let $k_{1}, \ldots, k_{s}$ be all the integers such that $k_{0} \geq k_{1}>\cdots>k_{s} \geq 1$ and $G^{m-k_{\ell}}(M) \subseteq$ $S^{m-k_{\ell}}(M)$ for $\ell=1, \ldots, s$. Clearly,

$$
\Gamma_{\ell}:=\left\{v \in H^{m-k_{\ell}}(M, \mathbb{Z} / 2) \mid\langle u \cup v,[M]\rangle=0 \text { for all } u \in A^{k_{\ell}}\right\}
$$

is a subgroup of $G^{m-k_{\ell}}(M)$. Choose an integer $d$ with $\operatorname{dim}_{\mathbb{Z} / 2} \Gamma_{\ell} \leq d$ for $\ell=1, \ldots, s$. Let

$$
B^{m-k_{\ell}}(d)=B^{m-k_{\ell}} \times \cdots \times B^{m-k_{\ell}} \quad \text { and } \quad B_{0}^{m-k_{\ell}}=B_{0}^{m-k_{\ell}} \times \cdots \times B_{0}^{m-k_{\ell}}
$$

be as in (2.2) (with $n=m-k_{\ell}$ ). Since every cohomology class in $\Gamma_{\ell}$ is spherical, there exists a smooth map $g_{\ell}=\left(g_{\ell 1}, \ldots, g_{\ell d}\right): M \rightarrow B^{m-k_{\ell}}(d)$ satisfying

$$
g_{\ell}(M) \subseteq B_{0}^{m-k_{\ell}}(d) \quad \text { and } \quad \Gamma_{\ell}=g_{\ell}^{*}\left(H^{m-k_{\ell}}\left(B^{m-k_{\ell}}(d), \mathbb{Z} / 2\right)\right) .
$$

Set

$$
\begin{gathered}
B=B^{m-k_{1}}(d) \times \cdots \times B^{m-k_{s}}(d), \quad B_{0}=B_{0}^{m-k_{1}}(d) \times \cdots \times B_{0}^{m-k_{s}}(d), \\
g=\left(g_{1}, \ldots, g_{s}\right): M \rightarrow B .
\end{gathered}
$$


Making use of Künneth's theorem and the inequalities $2\left(m-k_{\ell}\right) \geq 2\left(m-k_{0}\right) \geq m+1$ for $\ell=1, \ldots, s$, we get

$$
H^{q}(B, \mathbb{Z} / 2)=0 \quad \text { for } 0<q \leq m, q \notin\left\{m-k_{1}, \ldots, m-k_{s}\right\} .
$$

Künneth's theorem also implies

$$
\Gamma_{\ell}=g^{*}\left(H^{m-k_{\ell}}(B, \mathbb{Z} / 2)\right) \quad \text { for } 1 \leq \ell \leq s .
$$

Assertion 1. The restriction $g \mid N: N \rightarrow B$, where $N:=N_{1} \cup \cdots \cup N_{b}$, is null homotopic.

Clearly, it suffices to prove that for each pair of integers $(\ell, e)$ with $1 \leq \ell \leq s$ and $1 \leq e \leq d$, the map $h_{\ell e} \mid N: N \rightarrow B_{0}^{m-k_{\ell}}$ is null homotopic, where $h_{\ell e}: M \rightarrow B^{m-k_{\ell}}$ is defined by $h_{\ell e}(x)=g_{\ell e}(x)$ for all $x$ in $M$. Recall that $B_{0}^{m-k_{\ell}}$ is diffeomorphic to $S^{m-k_{\ell}}$. Let $\sigma$ be a generator of $H^{m-k_{\ell}}\left(B_{0}^{m-k_{\ell}}, \mathbb{Z}\right) \cong \mathbb{Z}$. Since $\operatorname{dim} N_{j} \leq m-k_{\ell}$ for $j=1, \ldots, b$, it follows from Hopf's classification theorem that $h_{\ell e} \mid N$ is null homotopic if and only if $\left(h_{\ell e} \mid N\right)^{*}(\sigma)=0$ in $H^{m-k_{\ell}}(N, \mathbb{Z})$. By the Mayer-Vietoris exact sequence, the last condition is equivalent to $\left(h_{\ell e} \mid N_{j}\right)^{*}(\sigma)=0$ in $H^{m-k_{\ell}}\left(N_{j}, \mathbb{Z}\right)$ for all $j=1, \ldots, b$.

If $\operatorname{dim} N_{j}<m-k_{\ell}$, then trivially $\left(h_{\ell e} \mid N_{j}\right)^{*}(\sigma)=0$.

Suppose that $\operatorname{dim} N_{j}=m-k_{\ell}$. In that case necessarily $\ell=1$ and $k_{1}=k_{0}$. In order to ease notation, set $h=h_{1 e}$. Since $N_{j}$ is connected and nonorientable, $\left(h \mid N_{j}\right)^{*}(\sigma)=0$ in $H^{m-k_{1}}\left(N_{j}, \mathbb{Z}\right)$ if and only if $\left(h \mid N_{j}\right)^{*}(\bar{\sigma})=0$ in $H^{m-k_{1}}\left(N_{j}, \mathbb{Z} / 2\right)$ where $\bar{\sigma}$ in $H^{m-k_{1}}\left(B_{0}^{m-k_{1}}, \mathbb{Z} / 2\right)$ is the reduction modulo 2 of $\sigma$. It follows from (4) that $h^{*}(\bar{\sigma})$ is in $\Gamma_{1}$, and hence (3) implies

$$
\left\langle h^{*}(\bar{\sigma}) \cup\left[N_{j}\right]^{M},[M]\right\rangle=0 .
$$

Therefore denoting by $\epsilon: N_{j} \hookrightarrow M$ the inclusion map, we have

$$
\begin{aligned}
\left\langle\left(h \mid N_{j}\right)^{*}(\bar{\sigma}),\left[N_{j}\right]\right\rangle & =\left\langle\epsilon^{*}\left(h^{*}(\bar{\sigma})\right),\left[N_{j}\right]\right\rangle=\left\langle h^{*}(\bar{\sigma}), \epsilon_{*}\left(\left[N_{j}\right]\right)\right\rangle=\left\langle h^{*}(\bar{\sigma}),\left[N_{j}\right]^{M} \cap[M]\right\rangle \\
& =\left\langle h^{*}(\bar{\sigma}) \cup\left[N_{j}\right]^{M},[M]\right\rangle=0 .
\end{aligned}
$$

Since $N_{j}$ is connected, we get $\left(h \mid N_{j}\right)^{*}(\bar{\sigma})=0$, as required. Assertion 1 is proved.

Choose a compact subset $K$ of $M$ such that $N$ is contained in the interior of $K$ and $N$ is a deformation retract of $K$, while $(M, K)$ is a polyhedral pair. Then $g \mid K: K \rightarrow B$ is null homotopic and, by the homotopy extension theorem [21, p. 118, Corollary 5], there exists a continuous map $g^{\prime}: M \rightarrow B$ which is homotopic to $g$ and $g^{\prime} \mid K$ is a constant map. Thus there is a smooth map $g^{\prime \prime}: M \rightarrow B$ homotopic to $g^{\prime}$ and equal to $g^{\prime}$ on $N$. Replacing, if necessary, $g$ by $g^{\prime \prime}$, we may assume that

$$
g: M \rightarrow B \text { is constant on } N=N_{1} \cup \cdots \cup N_{b},
$$

while (4) and (6) still hold.

Let $c: M \rightarrow B$ be a constant map sending $M$ to a point in $B_{0}$.

Assertion 2. The maps $(f, g): M \rightarrow G \times B$ and $(f, c): M \rightarrow G \times B$ represent the same bordism class in the unoriented bordism group $\mathcal{N}_{*}(G \times B)$. 
In view of Theorem 2.3 and Künneth's theorem, it suffices to prove that for every pair $(p, q)$ of nonnegative integers and all cohomology classes $\alpha$ in $H^{p}(G, \mathbb{Z} / 2)$ and $\beta$ in $H^{q}(B, \mathbb{Z} / 2)$, we have

$$
\begin{aligned}
\left\langle w_{i_{1}}(M) \cup \cdots \cup w_{i_{r}}(M) \cup\right. & \left.(f, g)^{*}(\alpha \times \beta),[M]\right\rangle \\
& =\left\langle w_{i_{1}}(M) \cup \cdots \cup w_{i_{r}}(M) \cup(f, c)^{*}(\alpha \times \beta),[M]\right\rangle
\end{aligned}
$$

for all nonnegative integers $i_{1}, \ldots, i_{r}$ with $i_{1}+\cdots+i_{r}=m-(p+q)$. Note that $(f, g)^{*}(\alpha \times \beta)=f^{*}(\alpha) \cup g^{*}(\beta)$ and $(f, c)^{*}(\alpha \times \beta)=f^{*}(\alpha) \cup c^{*}(\beta)$.

If $q=0$, then $g^{*}(\beta)=c^{*}(\beta)$, and hence (8) holds.

Suppose now $0<q \leq m$. Then $c^{*}(\beta)=0$ and (8) is equivalent to

$$
\left\langle w_{i_{1}}(M) \cup \cdots \cup w_{i_{r}}(M) \cup f^{*}(\alpha) \cup g^{*}(\beta),[M]\right\rangle=0 .
$$

If $q \notin\left\{m-k_{1}, \ldots, m-k_{s}\right\}$, then $\beta=0$ according to (4), and hence (9) holds. If $q=m-k_{\ell}$ for some $\ell$, then $g^{*}(\beta)$ is in $\Gamma_{\ell}$ in view of (5). Since (b) is satisfied, (2) implies that $w_{i_{1}}(M) \cup \cdots \cup w_{i_{r}}(M) \cup f^{*}(\alpha)$ is in $A^{k_{\ell}}$. Thus (9) holds in view of (3). Assertion 2 is proved.

The proof of Theorem 2.4 can be completed as follows. We may assume that $M$ is a Zariski closed nonsingular subvariety of $\mathbb{R}^{\mu}$ for some $\mu$. Then $N$, being a union of finitely many Zariski closed nonsingular subvarieties of $\mathbb{R}^{\mu}$, is a nice set, equivalently, a quasiregular subvariety, in the terminology used in [2] and [24], respectively (cf. [24, p. 75]). Since $(f, c)$ is a regular map, and by (7) the restriction $(f, g) \mid N$ is also regular, it follows from Assertion 2 that [2, Theorem 2.8.4] is applicable. Hence there exist a nonnegative integer $v$, a Zariski closed nonsingular subvariety $X$ of $\mathbb{R}^{\mu+v}$, a smooth diffeomorphism $\varphi: X \rightarrow M$, and a regular map $(\bar{f}, \bar{g}): X \rightarrow G \times B$ such that identifying $\mathbb{R}^{\mu}$ with $\mathbb{R}^{\mu} \times\{0\} \subseteq \mathbb{R}^{\mu+v}$, we have $N \subseteq X, \varphi(x)=x$ for all $x$ in $N$, and $(\bar{f}, \bar{g})$ is homotopic to $(f, g) \circ \varphi=(f \circ \varphi, g \circ \varphi)$. In particular, setting

$$
\begin{aligned}
& \bar{f}=\left(\bar{f}_{1}, \ldots, \bar{f}_{a}\right): X \rightarrow G=\mathbb{G}_{n, r_{1}} \times \cdots \times \mathbb{G}_{n, r_{a}}, \\
& \bar{g}=\left(\bar{g}_{1}, \ldots, \bar{g}_{s}\right): X \rightarrow B=B^{m-k_{1}}(d) \times \cdots \times B^{m-k_{s}}(d),
\end{aligned}
$$

we obtain $\bar{f}_{i}^{*}=\varphi^{*} \circ f_{i}^{*}$ and $\bar{g}_{\ell}^{*}=\varphi^{*} \circ g_{\ell}^{*}$ in cohomology for $1 \leq i \leq a$ and $1 \leq \ell \leq s$.

The cohomology class

$$
\varphi^{*}\left(f_{i}^{*}\left(w\left(\gamma_{n, r_{i}}\right)\right)\right)=\bar{f}_{i}^{*}\left(w\left(\gamma_{n, r_{i}}\right)\right)
$$

is in $H_{\mathrm{alg}}^{*}(X, \mathbb{Z} / 2)$, the map $\bar{f}_{i}$ being regular. Clearly,

$$
\varphi^{*}\left(\left[N_{j}\right]^{M}\right)=\left[N_{j}\right]^{X}
$$

is also in $H_{\mathrm{alg}}^{*}(X, \mathbb{Z} / 2)$. Hence (1) implies

$$
\varphi^{*}(A) \subseteq H_{\mathrm{alg}}^{*}(X, \mathbb{Z} / 2) .
$$


In particular,

$$
\varphi^{*}\left(A^{k_{\ell}}\right) \subseteq H_{\mathrm{alg}}^{k_{\ell}}(X, \mathbb{Z} / 2) \quad \text { for } \ell=1, \ldots, s .
$$

It remains to prove that the inclusion in (10) is actually an equality. By (2.2) and (4),

$$
\Gamma_{\ell}=g_{\ell}^{*}\left(\operatorname{Alg}^{m-k_{\ell}}\left(B^{m-k_{\ell}}(d)\right)\right),
$$

and hence

$$
\varphi\left(\Gamma_{\ell}\right)=\varphi^{*}\left(g_{\ell}^{*}\left(\operatorname{Alg}^{m-k_{\ell}}\left(B^{m-k_{\ell}}(d)\right)\right)\right)=\bar{g}_{\ell}^{*}\left(\operatorname{Alg}^{m-k_{\ell}}\left(B^{m-k_{\ell}}(d)\right)\right) .
$$

Consequently,

$$
\varphi^{*}\left(\Gamma_{\ell}\right) \subseteq \operatorname{Alg}^{m-k_{\ell}}(X),
$$

the map $\bar{g}_{\ell}: X \rightarrow B^{m-k_{\ell}}(d)$ being regular. By the Poincare duality,

$$
H^{k_{\ell}}(M, \mathbb{Z} / 2) \times H^{m-k_{\ell}}(M, \mathbb{Z} / 2) \rightarrow \mathbb{Z} / 2, \quad(u, v) \mapsto\langle u \cup v,[M]\rangle
$$

is a dual pairing, and therefore (3), (10), (11) and Theorem 2.1 taken together imply

$$
\varphi^{*}\left(A^{k_{\ell}}\right)=H_{\mathrm{alg}}^{k_{\ell}}(X, \mathbb{Z} / 2) \quad \text { for } \ell=1, \ldots, s,
$$

as required. The proof is complete.

We will need the following, purely technical, observation.

Lemma 2.5. Let $M$ be a compact connected smooth manifold of dimension $m$. Then:

(i) $G^{m-1}(M) \subseteq S^{m-1}(M)$ provided $m \geq 2$.

(ii) $G^{m-2}(M) \subseteq S^{m-2}(M)$ provided $m \geq 5$ and $H_{m-2}(M, \mathbb{Z})$ has no 2-torsion.

(iii) $G^{m-2}(M) \subseteq S^{m-2}(M)$ provided $m \geq 5, M$ is orientable, and $H_{1}(M, \mathbb{Z})$ has no 2-torsion.

(iv) $H^{m-3}(M, \mathbb{Z} / 2)=S^{m-3}(M)$ provided $m \geq 7, M$ is a spin manifold, and $H_{2}(M, \mathbb{Z})$ has no 2-torsion.

Proof. Given a smooth manifold $P$, we denote by $\tau_{P}$ its tangent bundle. The normal bundle of a smooth submanifold $N$ of $M$ will be denoted by $v_{N}$. Recall that $v_{N}$ is a trivial vector bundle if and only if $[N]^{M}$ is in $S^{k}(M), k=\operatorname{codim}_{M} N$.

(i) Let $u$ be in $G^{m-1}(M)$, that is, $\left\langle w_{1}(M) \cup u,[M]\right\rangle=0$. Since $M$ is connected, we have

$$
w_{1}(M) \cup u=0 .
$$

Choose a smooth connected curve $C$ in $M$ with $u=[C]^{M}$. It suffices to prove that the normal bundle $v_{C}$ is trivial or, equivalently, $w_{1}\left(v_{C}\right)=0$. Since $\tau_{C} \oplus v_{C}=\tau_{M} \mid C$ and $\tau_{C}$ is trivial, we have

$$
w_{1}\left(\nu_{C}\right)=w_{1}\left(\tau_{M} \mid C\right)=e^{*}\left(w_{1}(M)\right),
$$


where $e: C \hookrightarrow M$ is the inclusion map. A simple computation yields

$$
\begin{aligned}
e_{*}\left(e^{*}\left(w_{1}(M)\right) \cap[C]\right) & =w_{1}(M) \cap e_{*}([C])=w_{1}(M) \cap\left([C]^{M} \cap[M]\right) \\
& =\left(w_{1}(M) \cup[C]^{M}\right) \cap[M]=\left(w_{1}(M) \cup u\right) \cap[M]=0 .
\end{aligned}
$$

Since $C$ is connected, we get $e^{*}\left(w_{1}(M)\right) \cap[C]=0$, and hence $e^{*}\left(w_{1}(M)\right)=0$. Thus $w_{1}\left(v_{C}\right)=0$, as required.

(ii) By the universal coefficient theorem, the torsion subgroups of $H_{m-2}(M, \mathbb{Z})$ and $H^{m-1}(M, \mathbb{Z})$ are isomorphic, and hence $H^{m-1}(M, \mathbb{Z})$ has no 2-torsion. It follows from another version of the universal coefficient theorem that the reduction modulo 2 homomorphism $\rho: H^{m-2}(M, \mathbb{Z}) \rightarrow H^{m-2}(M, \mathbb{Z} / 2)$ is surjective.

By Wu's theorem [17. Theorem 11.14], the second Wu class of $M$ is equal to $w_{1}(M) \cup$ $w_{1}(M)+w_{2}(M)$, and consequently the Steenrod square

$$
\mathrm{Sq}^{2}: H^{m-2}(M, \mathbb{Z} / 2) \rightarrow H^{m}(M, \mathbb{Z} / 2)
$$

is given by $\operatorname{Sq}^{2}(u)=\left(w_{1}(M) \cup w_{1}(M)+w_{2}(M)\right) \cup u$. Therefore for $u$ in $G^{m-2}(M)$, we have $\left\langle\mathrm{Sq}^{2}(u),[M]\right\rangle=0$, which implies $\operatorname{Sq}^{2}(u)=0$, the manifold $M$ being connected. Since $\rho$ is surjective, Steenrod's classification theorem [21, p. 460, Theorem 15] implies that the cohomology class $u$ is spherical. Thus $u$ is in $S^{m-2}(M)$, and the proof of (ii) is complete.

(iii) By the universal coefficient theorem, the torsion subgroups of $H^{2}(M, \mathbb{Z})$ and $H_{1}(M, \mathbb{Z})$ are isomorphic. The Poincaré duality implies $H^{2}(M, \mathbb{Z}) \cong H_{m-2}(M, \mathbb{Z})$, and hence (iii) follows from (ii).

(iv) Since $H_{2}(M, \mathbb{Z})$ has no 2-torsion, the reduction modulo 2 homomorphism $H_{3}(M, \mathbb{Z}) \rightarrow H_{3}(M, \mathbb{Z} / 2)$ is surjective. Hence by Thom's theorem [22, Théorème II.27] each homology class in $H_{3}(M, \mathbb{Z} / 2)$ can be represented by an orientable smooth submanifold of $M$. It remains to prove that if $N$ is an orientable smooth submanifold of $M$ of dimension 3, then the normal bundle $v_{N}$ is trivial. The orientability of $N$ implies $w_{i}(N)=0$ for $i=1,2$. Since $\tau_{N} \oplus v_{N}=\tau_{M} \mid N$ and $M$ is a spin manifold, we get $w_{i}\left(v_{N}\right)=0$ for $i=1,2$. It follows from the last equality that $v_{N}$ is stably trivial (cf. for example [9. Lemma 1.2]). Finally, $v_{N}$ is trivial, since $\operatorname{rank} v_{N} \geq 4>3=\operatorname{dim} N$.

We are now ready to prove the results announced in Section 1.

Proof of Theorem 1.1. Every element of $H^{1}(M, \mathbb{Z} / 2)$ is of the form $w_{1}(\lambda)$ for some real line bundle $\lambda$ on $M$. Clearly

$$
w(\lambda)=1+w_{1}(\lambda)
$$

We claim that every element of $H^{2}(M, \mathbb{Z} / 2)$ is of the form $w_{2}(\xi)$ for some rank 2 real vector bundle $\xi$ on $M$ with $w_{1}(\xi)=0$. Indeed, by the universal coefficient theorem, the torsion subgroups of $H_{2}(M, \mathbb{Z})$ and $H^{3}(M, \mathbb{Z})$ are isomorphic. Hence $H^{3}(M, \mathbb{Z})$ has no 2-torsion, which implies that the reduction modulo 2 homomorphism $\rho: H^{2}(M, \mathbb{Z}) \rightarrow$ $H^{2}(M, \mathbb{Z} / 2)$ is surjective. Every element of $H^{2}(M, \mathbb{Z})$ is the first Chern class $c_{1}(\xi)$ of 
some complex line bundle $\xi$ on $M$. Regarding $\xi$ as a rank 2 real vector bundle, we get $w_{2}(\xi)=\rho\left(c_{1}(\xi)\right)$ and $w_{1}(\xi)=0$, which proves the claim. Note that

$$
w(\xi)=1+w_{2}(\xi) .
$$

Since $M$ is a spin manifold, we have $w_{i}(M)=0$ for $i=1,2,3$ (cf. [17, Problem 8-B]). Let $B$ be the subring of $H^{*}(M, \mathbb{Z} / 2)$ generated by $A$ and $w_{j}(M)$ for $j \geq 0$. Then $B$ is an admissible subring with

$$
A \subseteq B \quad \text { and } \quad A^{k}=B^{k} \quad \text { for } k=0,1,2,3 .
$$

In view of $(*)$ and $(* *)$, one can find a collection $\mathcal{F}$ of real vector bundles on $M$ and a collection $\mathcal{G}$ of smooth submanifolds of $M$ such that $B=A(\mathcal{F}, \mathcal{G})$ and $\operatorname{codim}_{M} N \geq 3$ for all $N$ in $\mathcal{G}$. By Theorem 2.4 and Lemma 2.5(i), (iii), (iv), there exist an algebraic model $X$ of $M$ and a smooth diffeomorphism $\varphi: X \rightarrow M$ satisfying

$$
\varphi^{*}(B) \subseteq H_{\mathrm{alg}}^{*}(X, \mathbb{Z} / 2) \quad \text { and } \quad \varphi^{*}\left(B^{k}\right)=H_{\mathrm{alg}}^{k}(X, \mathbb{Z} / 2) \quad \text { for } k=0,1,2,3 .
$$

The proof is complete.

Proof of Corollary 1.2. We first recall some results due to Thom [22]. Let $N$ be a compact $n$-dimensional manifold. By [22, Théorème II.26], every homology class in $H_{k}(N, \mathbb{Z} / 2)$ can be represented by a smooth submanifold, provided $2 k \leq n$ or $k=n-1$ or $(n, k)=$ $(7,4)$. If $N$ is orientable and $n \leq 9$, then according to [22, Corollaire II.28], every homology class in $H_{\ell}(N, \mathbb{Z}), \ell \geq 0$, can be represented by an oriented smooth submanifold.

We can now easily complete the proof. By the Poincare duality and the universal coefficient theorem, the reduction modulo 2 homomorphism $H_{p}(M, \mathbb{Z}) \rightarrow H_{p}(M, \mathbb{Z} / 2)$ is surjective in either of the following two cases:

(i) $m=7$ and $p=5$,

(ii) $m=8$ or 9 and $m / 2<p \leq m-2$.

Hence Thom's results recalled above imply that every homology class in $H_{k}(M, \mathbb{Z} / 2)$, $k \geq 0$, can be represented by a smooth submanifold. In particular, every subring of $H^{*}(M, \mathbb{Z} / 2)$ is admissible. The proof is complete in view of Theorem 1.1.

Proof of Theorem 1.3. We already recalled in the proof of Theorem 2.4 that $w(Y)$ is in $H^{*}(Y, \mathbb{Z} / 2)$ for every compact nonsingular real algebraic variety $Y$. Hence (a) implies (b).

Assume that (b) holds. By Lemma $2.5, G^{m-k}(M) \subseteq S^{m-k}(M)$ for $k=1$, 2. Since every element of $H^{1}(M, \mathbb{Z} / 2)$ is of the form $w_{1}(\lambda)$ for some real line bundle $\lambda$ on $M$ and since $w(\lambda)=1+w_{1}(\lambda)$, we have $A=A(\mathcal{F}, \mathcal{G})$, where $\mathcal{F}$ is a collection of real vector bundles on $M$ and $\mathcal{G}$ is a collection of smooth submanifolds of $M$ with $\operatorname{codim}_{M} N \geq 2$ for all $N$ in $\mathcal{G}$. It follows from Theorem 2.4 that (a) is satisfied.

We conclude this paper by examining consequences of Theorems 1.1 and 2.4 for the $n$ fold product $T^{n}=S^{1} \times \cdots \times S^{1}$. The interested reader will notice that there are other examples of a similar type. 
Example 2.6. Every homology class in $H_{p}\left(T^{n}, \mathbb{Z} / 2\right), \quad p \geq 0$, can be represented by a smooth submanifold, and hence every subring $A$ of $H^{*}\left(T^{n}, \mathbb{Z} / 2\right)$ is admissible. By Theorem 1.1, if $n \geq 7$, then there exist an algebraic model $X$ of $T^{n}$ and a smooth diffeomorphism $\varphi: X \rightarrow T^{n}$ satisfying

$$
\varphi^{*}(A) \subseteq H_{\mathrm{alg}}^{*}(X, \mathbb{Z} / 2) \quad \text { and } \quad \varphi^{*}\left(A^{k}\right)=H_{\mathrm{alg}}^{k}(X, \mathbb{Z} / 2) \quad \text { for } k=0,1,2,3 .
$$

Furthermore, for any $n \geq 1$, if $A$ is generated by 1 and some cohomology classes in $H^{i}\left(T^{n}, \mathbb{Z} / 2\right), i=1,2$, then $X$ and $\varphi$ can be chosen in such a way that

$$
\varphi^{*}(A) \subseteq H_{\mathrm{alg}}^{*}(X, \mathbb{Z} / 2) \quad \text { and } \quad \varphi^{*}\left(A^{k}\right)=H_{\mathrm{alg}}^{k}(X, \mathbb{Z} / 2) \quad \text { for } 2 k+1 \leq n .
$$

Indeed, one readily checks that $A=A(\mathcal{F})$, where $\mathcal{F}$ is a collection of real vector bundles on $T^{n}$. Since $H^{m-k}\left(T^{n}, \mathbb{Z} / 2\right)=S^{m-k}\left(T^{n}\right)$ for all $k$ with $2 k+1 \leq n$, the existence of $X$ and $\varphi$ satisfying the required properties is guaranteed by Theorem 2.4 .

Acknowledgments. The paper was completed at the Max-Planck-Institut für Mathematik in Bonn, whose support and hospitality are gratefully acknowledged.

\section{References}

[1] Akbulut, S., King, H.: The topology of real algebraic sets. Enseign. Math. 29, 221-261 (1983) Zbl 0541.14019 MR 0719311

[2] Akbulut, S., King, H.: Topology of Real Algebraic Sets. Math. Sci. Res. Inst. Publ. 25, Springer (1992). Zbl 0808.14045 MR 1225577

[3] Benedetti, R., Dedò, M.: Counterexamples to representing homology classes by real algebraic subvarieties up to homeomorphism. Compos. Math. 53, 143-151 (1984) Zbl 0547.14019 MR 0766294

[4] Benedetti, R., Tognoli, A.: Approximation theorems in real algebraic geometry. Boll. Un. Mat. Ital. Suppl. 1980, no. 2, 209-228 Zbl 0465.14011 MR 0675502

[5] Benedetti, R., Tognoli, A.: On real algebraic vector bundles. Bull. Sci. Math. (2) 104, 89-112 (1980) Zbl 0421.58001 MR 0560747

[6] Benedetti, R., Tognoli, A.: Remarks and counterexamples in the theory of real vector bundles and cycles. In: Géométrie algébrique réelle et formes quadratiques, Lecture Notes in Math. 959, Springer, 198-211 (1982) Zbl 0498.14015 MR 0683134

[7] Bochnak, J., Coste, M., Roy, M.-F.: Real Algebraic Geometry. Ergeb. Math. Grenzgeb. 36, Springer, Berlin (1998) Zbl 0912.14023 MR 1659509

[8] Bochnak, J., Kucharz, W.: Algebraic models of smooth manifolds. Invent. Math. 97, 585-611 (1989) Zbl 0687.14023 MR 1005007

[9] Bochnak, J., Kucharz, W.: K-theory of real algebraic surfaces and threefolds. Math. Proc. Cambridge Philos. Soc. 106, 471-480 (1989) Zbl 0707.14006 MR 1010372

[10] Bochnak, J., Kucharz, W.: Algebraic cycles and approximation theorems in real algebraic geometry. Trans. Amer. Math. Soc. 337, 463-472 (1993) Zbl 0809.57015 MR 1091703

[11] Bochnak, J., Kucharz, W.: On homology classes represented by real algebraic varieties. In: Singularities Symposium—- Łojasiewicz 70, Banach Center Publ. 44, Inst. Math., Polish Acad. Sci., 21-35 (1998) Zbl 0915.14033 MR 1677394

[12] Borel, A., Haefliger, A.: La classe d'homologie fondamentale d'un espace analytique. Bull. Soc. Math. France 89, 461-513 (1961) Zbl 0102.38502 MR 0149503 
[13] Conner, P. E.: Differentiable Periodic Maps. 2nd ed., Lecture Notes in Math. 738, Springer (1979) Zbl 0417.57019 MR 0548463

[14] Kucharz, W.: On homology of real algebraic sets. Invent. Math. 82, 19-26 (1985) Zbl 0547.14018 MR 0808106

[15] Kucharz, W.: Algebraic equivalence and homology classes of real algebraic cycles. Math. Nachr. 180, 135-140 (1996) Zbl 0877.14003 MR 1397672

[16] Kucharz, W.: Algebraic cycles and algebraic models of smooth manifolds. J. Algebraic Geom. 11, 101-127 (2002) Zbl 1060.14084 MR 1865915

[17] Milnor, J., Stasheff, J.: Characteristic Classes. Ann. of Math. Stud. 76, Princeton Univ. Press, Princeton, NJ (1974) Zbl 0298.57008 MR 0440554

[18] Nash, J.: Real algebraic manifolds. Ann. of Math. 56, 405-421 (1952) Zbl 0048.38501 MR 0050928

[19] Risler, J.-J.: Sur l'homologie des surfaces algébriques réelles. In: Géométrie algébrique réelle et formes quadratiques, Lecture Notes in Math. 959, Springer, 381-385 (1982) Zbl 0503.14014 MR 0683144

[20] Silhol, R.: A bound on the order of $H_{n-1}^{(a)}(X, \mathbb{Z} / 2)$ on a real algebraic variety. In: Géometrie algébrique réelle et formes quadratiques, Lecture Notes in Math. 959, Springer, 443-450 (1982) Zbl 0558.14003 MR 0683148

[21] Spanier, E.: Algebraic Topology. Springer, New York (1966) Zbl 0810.55001 MR 1325242

[22] Thom, R.: Quelques propriétés globales de variétés différentiables. Comment. Math. Helv. 28, 17-86 (1954) Zbl 0057.15502 MR 0061823

[23] Tognoli, A.: Su una congettura di Nash. Ann. Scuola Norm. Sup. Pisa Sci. Fis. Mat. (3) 27, 167-185 (1973) Zbl 0263.57011 MR 0396571

[24] Tognoli, A.: Algebraic approximation of manifolds and spaces. In: Séminaire Bourbaki, 32e année, 1979/1980, no. 548, Lecture Notes in Math. 842, Springer, $73-94$ (1981) Zbl 0456.57012 MR 0636518 